ORIGINAL RESEARCH ARTICLE

\title{
Service Availability and Readiness Assessment of Maternal, Newborn and Child Health Services at Public Health Facilities in Madagascar
}

\author{
Sandrine H. Andriantsimietry ${ }^{1}$, Raymond Rakotomanga ${ }^{2}$, Jean Pierre Rakotovao ${ }^{1}$, Eliane \\ Ramiandrison ${ }^{1}$, Marc Eric R. Razakariasy', Rachel Favero ${ }^{2}$, Patricia Gomez ${ }^{2}$, Blami Dao ${ }^{2}$ and \\ Eva Bazant ${ }^{2}$
}

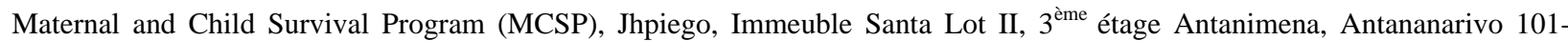
Madagascar ${ }^{1}$; Jhpiego, 1615 Thames Street, Baltimore, MD 21231, USA ${ }^{2}$

*For Correspondence: Email: sandrine.andriantsimietry@jhpiego.org, Phone: +261 340263283.

\begin{abstract}
The Service Availability and Readiness Assessment (SARA) survey was adapted and used to generate information on service availability and the readiness of maternal, newborn and child health facilities to provide basic health care interventions for obstetric care, neonatal and child health in Madagascar. The survey collected data from fifty-two public health facilities, ranging from university hospitals (CHU), referral district and regional hospitals (CHD/ CHRR) to basic health centres (CSB). For basic emergency obstetric and newborn care (BEmONC) readiness, on average, CHU had nine (71.8\%), CHD/CHRR had eight and CSB had six out of the thirteen tracer items. Regarding the availability of the eleven tracer items for comprehensive CEmONC services, on average a CHU had nine ( $80.0 \%$ ), a CHRR had eight $(71.1 \%)$ and a CHD that is the only type of hospitals in rural area had three tracer items (30.0\%). Tracer item availability results are low, indicating the need to strengthen supplies at basic health centers in order to improve the chances of success of Madagascar's Roadmap for accelerating the reduction of the maternal and neonatal mortality 2015-2019, and meeting Sustainable Development Goals 3.1 and 3.2. (Afr J Reprod Health 2016 (Special Edition); 20[3]: 149-158).
\end{abstract}

Keywords: Madagascar, Maternal and Child health services, Service availability and readiness assessment, Public health facilities

\section{Résumé}

L'Enquête sur la disponibilité du service et l'évaluation de l'état de préparation des services de santématernelle, néonatale et infantile (DSEP) a été adaptée et utilisée pour générer des informations sur la disponibilité du service et la disponibilité des établissements de santé maternelle, néonatale et infantile 'à assurer des interventions de soins de santé de base pour les soins obstétriques, la santé néonatale et infantile à Madagascar. L'enquête a recueilli des données à partir de cinquante-deux établissements de santé publique, couvrant des Centres hospitaliers universitaires (CHU), Centres d'orientation du District et les hôpitaux régionaux d'orientation (CHD / CHRR) aux centres de santé primaire(CSB). Pour les soins obstétricaux d'urgence de base et les soins du nouveau-né (SONUB) la préparation, en moyenne, CHU avait neuf (71,8\%), CHD / CHRR avait huit et CSB avait six sur treize articles de traçage. En ce qui concerne la disponibilité des onze éléments traceurs pour les services SONUC complets, en moyenne, un CHU en avait neuf (80,0\%), un CHRR en avait huit $(71,1 \%)$ et qui est le seul type d'hôpitaux dans la région rurale avait trois éléments traceurs $(30,0 \%)$ les résultats de la disponibilité des éléments traceurs sont faibles, ce qui indique la nécessité de renforcer l' approvisionnement des centres de santé primaire afin d'améliorer les possibilités de succès de la feuille de route de Madagascar pour accélérer la réduction de la mortalité maternelle et néonatale 2015-2019, et pour accomplir des objectifs de développement durable 3.1 et 3.2. (Afr J Reprod Health 2016 (Edition Spéciale); 20[3]: 149-158).

Mots-clés: Madagascar, services de santé maternelle et infantile, disponibilité du service et l'évaluation de l'état de préparation, établissements de santé publics.

\section{Introduction}

In many countries, insufficient progress was made towards Millennium Development Goals MDG 4 (reduction of child mortality) and MDG 5 (reduction of maternal mortality) ${ }^{1,2}$ and the current Sustainable Development Goals (SDG) 3.1 and $3.2^{3}$. Despite increased investment in maternal, newborn and child health ${ }^{4}$, coverage of effective interventions for both direct and indirect causes of deaths is far from universal and must be improved to end all preventable maternal, newborn and child deaths ${ }^{5}$. Most maternal and newborn deaths occur on the day of birth, therefore essential lifesaving interventions need to be delivered at facilities with capacity to provide support of normal labor and 
birth as well as basic or comprehensive emergency obstetric and newborn care (BEmONC or CEmONC) $)^{6,7,8}$. ANC services are a platform for preventing and/or treating conditions such as tetanus, syphilis, HIV, malaria, anemia and hypertensive disorders which lead to poor outcomes for both mother and newborn. In ANC, women receive counseling about birth preparedness, complication readiness and skilled care at birth ${ }^{9}$. A useful output indicator for monitoring supply-side progress towards sufficient services for reducing maternal mortality is the availability of facilities capable of providing lifesaving EmONC ${ }^{10,11}$.

More than half of maternal deaths globally occur in sub-Saharan Africa ${ }^{12}$. It is evident that global targets will not be met due to the limited progress made toward the Millennium Development Goals in this region ${ }^{13}$.

In Madagascar, the mortality rate among children under 12 months is 42/1000 live births but newborn deaths account for nearly $50 \%$ of these and must be reduced to increase overall survival among children under five years old. The maternal mortality ratio has remained unchanged over two decades and is at 478 maternal deaths per 100,000 live births. Therefore, the MDG 5 target of 122 maternal deaths/100,000 live births was not met in $2015^{14}$

In 2010, only 22 facilities out of 294 visited in the national EmONC survey were found to offer EmONC. Considering the UN process indicator of one $\mathrm{CEmONC}$ and four BEmONC facilities per 500,000 population, the finding revealed a need to establish twenty new CEmONC and 172 BEmONC sites ${ }^{1516}$. Some good news is that Madagascar is among the six low income African countries that have achieved a neonatal mortality of 26 per 1,000 live births despite a gross national income per capita under US400 per year ${ }^{9}$.

To inform the design of effective interventions to reduce maternal, newborn and child mortality and morbidity in Madagascar, the United States Agency for International Development (USAID)-supported Maternal and Child Survival Program (MCSP) ${ }^{17}$ carried out a Service Availability and Readiness Assessment $(\text { SARA })^{18}$ in public health centers in the fifteen USAID intervention regions during its start-up phase. SARA survey is part of strong country monitoring system of facilities and readiness to deliver services, and track how health systems respond to increased inputs and improved outputs and impact on health systems. Therefore, its results can indicate how Madagascar is performing in relation to achieving MDGs/SDGs. This manuscript presents the assessment's findings for $\mathrm{ANC}, \mathrm{BEmONC}$ and $\mathrm{CEmONC}$, and will inform the new Ministry of Health-sponsored Roadmap Strategy for Acceleration of the Reduction of Maternal and Newborn Mortality for the next five years.

\section{Methods}

The purpose of SARA was to establish baseline data prior to provision of technical support to the Ministry of Health. MCSP's aim is to improve key maternal, newborn and child health outcomes through delivery of high-quality basic health care services.

\section{Setting}

The assessment was conducted in fifteen of the twenty-two regions in Madagascar, which comprise $77.9 \%$ of the total population ${ }^{19}$ : Alaotra Mangoro, Amoron'i Mania, Analamanga, Atsinanana, Atsimo Andrefana, Boeny, Diana, Haute Matsiatra, Ihorombe, Melaky, Menabe, Sofia, Sava, Vakinankaratra, Vatovavy Fitovinany.

\section{Design, sampling}

This cross-sectional readiness assessment of maternal, newborn and child health was carried out in public health facilities of all types, which are state-mandated to provide maternal, newborn and child health services. The sampling frame for this study included a list of public basic health centers (Centre de Santé de Base, CSB) that are part of the two USAID bilaterals JSI/MAHEFA ${ }^{20}$ and

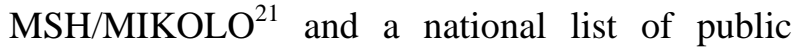
hospitals. We chose two CSBs, one referral hospital and one university hospital per region to assess. Only six of these regions have a university hospital (Centre Hospitalier Universitaire, CHU). For each of these six regions, one referral hospital at district level (Centre Hospitalier du District, 
CHD) was included; and for the remaining regions, one referral hospital at regional level (Centre Hospitalier de Référence Régional, CHRR) was selected for the assessment. The CSB selection criterion was less than two-hour travel distance from a referral hospital. An exception was made for Boeny and Atsinanana regions where an extra CSB was added, to replace the CHD in Boeny and to replace a CSB closed during the days of the assessment in Atsinanana. Thus, a representative sample of 52 public health facilities which included six CHUs, 14 referral hospitals (nine CHRR and five CHD) and 32 CSBs was assessed (Table 1).

Table 1: Characteristics of the Health Facilities Assessed. Madagascar, 2014.

\begin{tabular}{llll}
\hline Facility level & $\begin{array}{l}\text { Referral } \\
\text { level }\end{array}$ & $\begin{array}{l}\text { Total in } \\
\text { the 15 } \\
\text { regions }\end{array}$ & $\begin{array}{l}\text { Total } \\
\text { selected } \\
\text { for } \\
\text { assessment }\end{array}$ \\
\hline $\begin{array}{l}\text { Basic Health } \\
\text { Centers (Centre } \\
\text { de Santé de Base- }\end{array}$ & $\begin{array}{l}\text { applicable } \\
\text { CSB) }\end{array}$ & 1,227 & $32(2.5 \%)$ \\
$\begin{array}{l}\text { District referral } \\
\text { hospitals First level }\end{array}$ & 124 & $5(4.0 \%)$ \\
$\begin{array}{l}\text { Hospitalier de } \\
\text { District- CHD) } \\
\text { Regional referral } \\
\text { hospitals (Centre }\end{array}$ & Second & 13 & \\
$\begin{array}{l}\text { Hospitalier de } \\
\text { Référence }\end{array}$ & & & \\
$\begin{array}{l}\text { Régionale- } \\
\text { CHRR) }\end{array}$ & & & \\
$\begin{array}{l}\text { University } \\
\text { teaching) }\end{array}$ & Third & 16 & \\
hospitals (Centre & & & \\
Hospitalier & & & \\
Universitaire- & & & \\
CHU) & & & \\
\hline
\end{tabular}

\section{Procedure}

\section{Adaptation of SARA tool}

WHO's SARA too ${ }^{18}$ is a comprehensive approach to evaluate areas including maternal, newborn and child health, and is used widely by global programs and donors, such as GAVI Alliance and Global Fund ${ }^{22}$ and is then a reliable standard tool to monitor health facilities and services delivery. The SARA instrument was adapted to focus mainly on service specific readiness and availability for maternal and newborn health. The presence of thirteen life-saving commodities ${ }^{24}$ including medicines, medical devices and health supplies for maternal, newborn and child health was assessed through the general service readiness in essential medicines and through integrated management of childhood illness (IMCI) readiness within our SARA adapted tool. As the assessment of commodities related to IMCI was an addendum to the assessment during the data collection period, zinc and oral rehydration solution (ORS) availability was assessed in 23 out of 32 CSB $(72 \%)$.

\section{Data collection and assessors}

The assessment took six hours on average at each facility and comprised health worker interviews, observation of availability and functionality of equipment and availability of supplies, medicines and commodities. Nine teams of one doctor supervisor and three midwives were trained in use of the tool and performed the survey in all health facilities sampled during three weeks in September 2014. Data were collected both on tablets using the CommCare Open Data Kit (USA) ${ }^{23}$ to facilitate the availability of an electronic database and on paper forms to verify the accuracy of the electronic data. Data entered into tablets by the field teams were uploaded to a central cloud server and automatically produced spreadsheets using Excel (Microsoft, Redmond, WA, USA).

\section{Data analysis}

At the end of the data collection period, data were merged and analyzed descriptively for each facility type using SPSS20 (SPSS Inc. Chicago, IL, USA) and Microsoft Excel for results tabulation and graphing.

Service readiness analyses described availability of essential inputs needed, called tracer items, to deliver service-specific interventions across five areas: (i) trained staff and (ii) relevant, up-to-date guidelines; (iii) functioning equipment; (iv) diagnostic capacities and (v) essential medicines and commodities ${ }^{22}$. Within each area a mean score was calculated and tracer items were given equal weight ${ }^{22}$.

\section{Ethics}

The Madagascar ethical committee determined that this readiness assessment of health facilities 
Figure 1: Geographical Distribution of Health Facilities Surveyed. Madagascar, 2014.

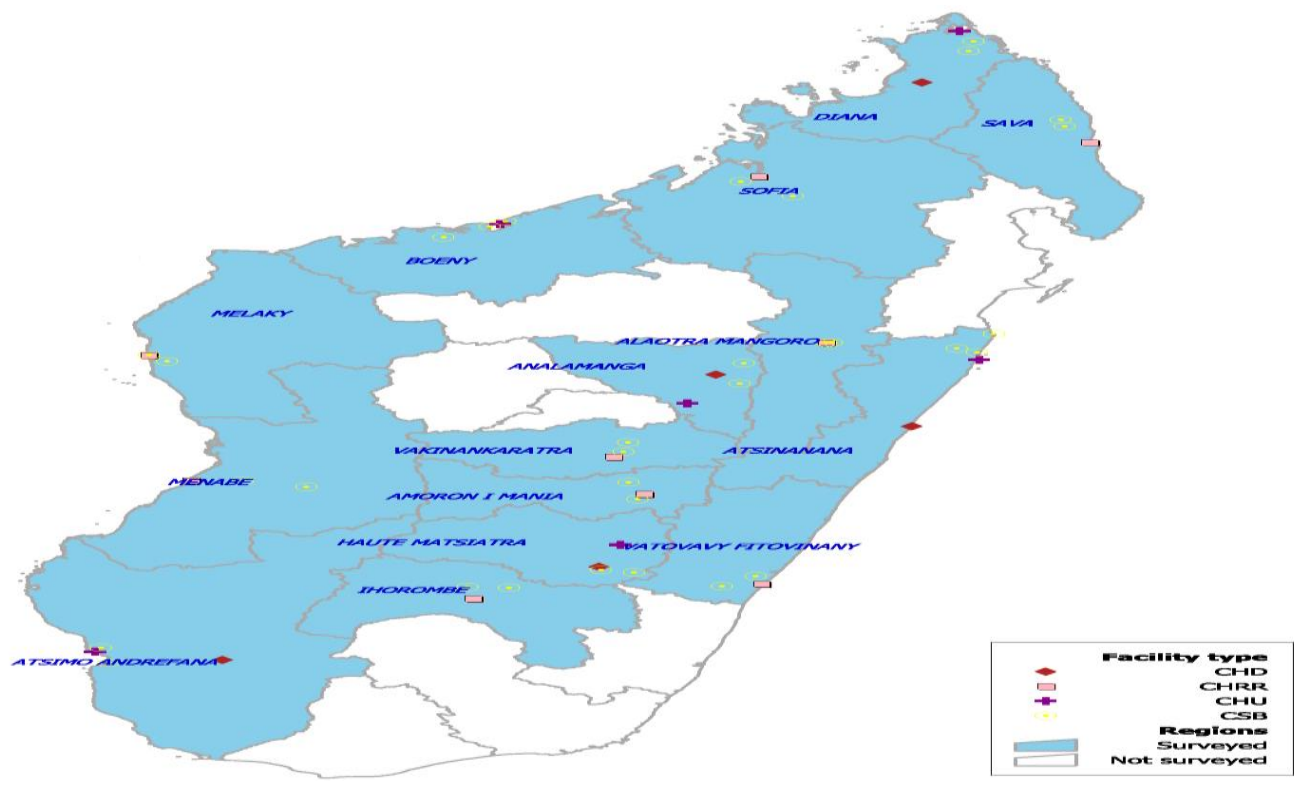

did not meet the criteria for human subject research and thus approval by them was not necessary. The implementing partner's institution in the Unites States also determined that this assessment did not meet the criteria for human subject research and therefore this assessment was not submitted to the Institutional Review Board. The Ministry of Health authorized this assessment in public facilities prior to the start of the assessment.

\section{Results}

Of the 52 health facilities, $12 \%$ were tertiary carelevel CHUs, $27 \%$ were referral-level hospitals (17\% CHRR and 10\% CHD), and 62\% were primary care level CSBs. The geographical location of the health facilities is shown in Figure 1.Readiness in delivery services focused on the presence of trained staff, guidelines, equipment and supplies, diagnostics and medicines and commodities.

\section{Antenatal care services}

On average, university hospitals had six of the ten ANC tracer items, for an ANC mean readiness score of $60.0 \%, \mathrm{CI}_{95 \%}: 31.8-88.2 \%$ (Table 2). The mean score was slightly lower in other facility types at $55.0 \%, \mathrm{CI}_{95 \%}: 41.0-69.0 \%$ for referral hospitals and 48.9\%, $\mathrm{CI}_{95 \%}: 34.5-63.3 \%$ for primary care facilities.

Availability of health providers working in antenatal care who had received technical updates in ANC over the last two years was low. Very few providers had received recent ANC training (16.7\% at $\mathrm{CHU}, 7.0 \%$ at referral hospitals and $9.4 \%$ at CSBs). Functioning blood pressure apparatus was relatively highly available in $83.3 \%$ of CHUs, $78.6 \%$ of CHD/CHRR and $84.4 \%$ of CSB.

\section{Basic emergency obstetric and newborn care services}

Of the seven BEmONC (parenteral treatment of maternal infection, pre-eclampsia/eclampsia and postpartum hemorrhage; removal of retained products of conception; assisted vaginal delivery; manual removal of placenta and newborn resuscitation) functions in the health facilities (Table 3), magnesium sulfate was available in the majority of referral hospitals $(57.1 \%)$, but in few CHUs (16.7\%) and in basic health centers $(6.3 \%)$. On the other hand, injectable antibiotics were less likely to be found at referral hospitals $(57.1 \%)$, and were universally available at CHUs (100\%) and widely available at CSBs $(81.3 \%)$. Emergency transport was available at $83.3 \%$ of CHUs, $64.3 \%$ of referral hospitals and exceptionally only one of 
Table 2: Percentage of Health Facilities Equipped with Tracer Items for Antenatal Care Services Among Facilities Providing this Service. Madagascar, 2014.

\begin{tabular}{llll}
\hline & CHU & CHD/ CHRR & CSB \\
\hline Tracer Item & $\mathbf{N = 6}$ & $\mathbf{N = 1 4}$ & $\mathbf{N = 3 2}$ \\
Trained staff & 16.7 & 7.0 & 9.4 \\
Guidelines & 0.0 & 50.0 & 12.5 \\
Job aids & 50.0 & 36.0 & 25.0 \\
Blood Pressure apparatus & 83.3 & 78.6 & 84.4 \\
Iron \& Folic Acid tablets & 50.0 & 50.0 & 78.1 \\
Urine dipstick protein & 100.0 & 85.7 & 5.0 \\
Haemoglobin test & 83.3 & 71.4 & 0.0 \\
Testing kits for syphilis & 83.3 & 78.6 & 93.8 \\
Testing kits for HIV & 100.0 & 71.4 & 81.3 \\
Tetanus toxoid vaccine & 33.3 & 21.0 & 100.0 \\
All 10 items & 0.0 & 0.0 & 0.0 \\
Mean Score [CI 95\%] & $\mathbf{6 0 . 0}[\mathbf{3 1 . 8 - 8 8 . 2 ]}$ & $\mathbf{5 5 . 0}[\mathbf{4 1 . 0} \mathbf{- 6 9 . 0}]$ & $\mathbf{4 8 . 9}[\mathbf{3 4 . 5 - 6 3 . 3}]$ \\
\hline
\end{tabular}

Table 3. Percentage of Health Facilities Equipped with Tracer Items for BEmONC Services Among Facilities Providing Delivery Service. Madagascar, 2014.

\begin{tabular}{llll}
\hline & CHU (\%) & CHD/ CHRR (\%) & CSB (\%) \\
\hline Tracer Item & $\mathbf{N = 6}$ & $\mathbf{N = 1 4}$ & $\mathbf{N}=\mathbf{3 2}$ \\
Trained staff BEmONC & 50.0 & 57.1 & 0.0 \\
Guidelines BEmONC & 33.3 & 35.7 & 0.0 \\
Emergency Transport & 83.3 & 64.3 & 3.1 \\
Neonatal bag and mask & 50.0 & 14.3 & 15.6 \\
Suction apparatus & 66.7 & 42.9 & 12.5 \\
Manual vacuum extractor & 83.3 & 78.6 & 71.9 \\
Vacuum aspirator/ D\&C kit & 100.0 & 64.3 & 71.9 \\
Magnesium sulfate & 16.7 & 57.1 & 6.3 \\
Injectable antibiotic & 100.0 & 57.1 & 81.3 \\
Skin disinfectant & 100.0 & 85.7 & 93.8 \\
Diazepam & 83.3 & 78.6 & 56.3 \\
Injectable uterotonic & 66.7 & 64.3 & 78.1 \\
Intravenous solution & 100.0 & 85.7 & 87.5 \\
All 13 items & 0.0 & 0.0 & 0.0 \\
Mean Score [CI 95\%] & $\mathbf{7 1 . 8}[\mathbf{5 8 . 0 - 9 0 . 8}]$ & $\mathbf{6 0 . 4}[\mathbf{4 6 . 3 - 9 7 . 9 ]}$ & $\mathbf{4 4 . 5}$ [32.0-58.4] \\
\hline
\end{tabular}

$32 \mathrm{CSBs}$, due to its location in a tourist area. Mean availability of tracer items for BEmONC was $71.8 \%$ at university hospitals, $60.4 \%$ at referral hospitals and $44.5 \%$ at basic health centers. None of the health facilities had all the tracer items available on site.

\section{Comprehensive emergency obstetric and newborn care services}

No hospitals had all ten CEmONC tracer items assessed to provide the nine functions of the CEmONC services (seven BEmONC functions plus availability of surgical capacity and blood transfusion) (Table 4). Functional anesthesia equipment was rare at district referral hospitals (20\%) but was available at the majority of regional referral hospitals $(55.5 \%)$. Sixty percent of these hospitals reported having trained staff in anesthesia and surgery. Blood sufficiency, defined as no interruption in blood availability in the past three months, was reported at half of university hospitals and $44.4 \%$ of regional referral hospitals, all of which offered caesarean sections. On the other hand, no district referral hospital had sufficient blood for CEmONC services. Furthermore, CEmONC guidelines were not found in any facility even though the staff reported to have received recent $\mathrm{CEmONC}$ training at $\mathrm{CHU}$ and CHRR. At regional hospitals, over two-thirds of providers $(66.7 \%)$ had received technical updates in CEmONC over the past two years and half of the $\mathrm{CHU}$ health providers had received them. However, few CHD providers $(20.0 \%)$ had received $\mathrm{CEmONC}$ technical update. An incubator was seen in $66.7 \%$ of $\mathrm{CHU}$ and $22.2 \%$ of CHRR and in none of the CHD. 
Table 4: Percentage of Health Facilities Equipped with Tracer Items for CEmONC Services Among Facilities Providing Delivery Service. Madagascar, 2014.

\begin{tabular}{|c|c|c|c|}
\hline & CHU (\%) & CHRR (\%) & CHD (\%) \\
\hline Tracer Item & $\mathrm{N}=6$ & $\mathrm{~N}=9$ & $\mathrm{~N}=5$ \\
\hline $\begin{array}{l}\text { Trained staff } \\
\text { CEmONC }\end{array}$ & 50.0 & 66.7 & 20.0 \\
\hline $\begin{array}{l}\text { Trained staff } \\
\text { surgery }\end{array}$ & 100.0 & 88.9 & 60.0 \\
\hline $\begin{array}{l}\text { Trained staff } \\
\text { anesthesia }\end{array}$ & 100.0 & 100.0 & 60.0 \\
\hline $\begin{array}{l}\text { Guidelines } \\
\text { CEmONC }\end{array}$ & 33.3 & 33.3 & 20.0 \\
\hline Incubator & 66.7 & 22.2 & 0 \\
\hline $\begin{array}{l}\text { Anesthesia } \\
\text { equipment }\end{array}$ & 100.0 & 55.5 & 20.0 \\
\hline Blood typing & 100.0 & 100.0 & 40.0 \\
\hline Cross match & 100.0 & 100.0 & 40.0 \\
\hline Blood safety & 50.0 & 44.4 & 0 \\
\hline $\begin{array}{l}\text { Blood } \\
\text { sufficiency }\end{array}$ & 100.0 & 100.0 & 40.0 \\
\hline All 11 items & 0 & 0 & 0 \\
\hline $\begin{array}{l}\text { Mean Score } \\
\text { [CI 95\%] }\end{array}$ & $\begin{array}{l}80.0[58.4- \\
100]\end{array}$ & $\begin{array}{r}71.1 \\
91.1]\end{array}$ & $\begin{array}{l}30.0 \text { [11.1- } \\
48.9]\end{array}$ \\
\hline
\end{tabular}

\section{Life-saving commodities in maternal, newborn and child health}

Availability of the thirteen life-saving commodities within the university hospitals, the first referral hospitals and the basic health centers is shown in Table 5. Data on thirteen life-saving tracer items was comprehensive for 23 out of 32 basic health facilities. The most available commodity differed for each facility type: newborn resuscitation devices $(83.3 \%)$ for $\mathrm{CHU}$, amoxicillin for CHD/CHRR (71.4\%) and CSB $(84.4 \%)$. Wide variation in availability of newborn resuscitation devices that include the newborn bag and mask and a resuscitation area was observed across the three facility types: $83 \%$ for university hospitals, $57.1 \%$ for referral hospitals and only $31.3 \%$ for basic health centers. In contrast, amoxicillin was present at similar levels across facility types: $84 \%$ of basic health centers, $71.4 \%$ of referral hospitals and $66.7 \%$ of CSBs had amoxicillin for treatment of sick children. Although misoprostol was available in only $7.1 \%$ of referral hospitals that were among the pilot sites for the introduction of misoprostol in Madagascar, injectable uterotonics were available in at least two thirds of all facilities surveyed (CHU: $66.7 \%$,
CHD/CHRR: $\quad 64.3 \%$ and CSB: $78.1 \%$ ). Chlorhexidine for umbilical cord care was available only in $6.3 \%$ of CSBs - the facilities that piloted the introduction of chlorhexidine for newborn care across the country. At CSBs, 52.2\% had zinc and $47.8 \%$ had ORS available on site during the assessment.

\section{Discussion}

This cross-sectional readiness assessment examined the status of maternal, newborn and child health services in public health facilities. An adaptation of WHO's SARA tool was used in 52 public health facilities in fifteen regions in the country.

\section{Antenatal care services}

Availability of antenatal care tracer items across all facilities is on average around 55\%. In other words, on average each facility type had half of the ten antenatal care tracer items available on site during the assessment. This finding is consistent with a previous study in the country, in particular for availability of antenatal care tracer items such as functional blood pressure apparatus, iron and folic acid and ANC guidelines ${ }^{25}$. However a slight improvement in availability of tetanus toxoid vaccine in primary care facilities was noted over the past four years between these surveys $(91 \%$ in 2010 vs $100 \%$ in 2014). Antenatal care providers should check blood pressure at each ANC visit as hypertensive disorders of pregnancy are a major cause of maternal and perinatal morbidity and mortality $^{5,}{ }^{26}$. In Madagascar, measuring blood pressure during antenatal care visits is relatively high $(82 \%)$ but performed correctly in only $28 \%$ according to the previous quality of care assessment in $2010^{25,27}$. This could be a consequence of lack of ANC national guidelines seen across health facilities as reported previously $^{25}$, in our survey. Availability of staff trained in antenatal care services and in EmONC in the last two years was generally low possibly due to the socio-political crisis in the country over the past five years. We found also that none of the hospitals surveyed had all the tracer items for 
Table 5: Percentage of Facilities Having 13 Life-Saving Commodities According to the UN Commission Guidance. Madagascar, 2014.

\begin{tabular}{lllll}
\hline Commodity by life stage & & CHU (\%) & CHD/ CHRR (\%) & CSB (\%) \\
\hline & & $\mathbf{N}=\mathbf{6}$ & $\mathbf{N = 1 4}$ & $\mathbf{N = 3 2}$ \\
\multirow{4}{*}{ Reproductive Health } & 33.3 & 14.3 & 21.9 \\
& Female condoms & 66.7 & 35.7 & 68.8 \\
& Implants & 50.0 & 21.4 & 15.6 \\
Maternal health & Emergency contraception & 66.7 & 64.3 & 78.1 \\
& Oxytocin & 16.7 & 57.1 & 6.3 \\
& Magnesium sulfate & 0.0 & 7.1 & 0.0 \\
Newborn Health & Misoprostol & 50.0 & 50.0 & 43.8 \\
& Injectable antibiotic & 0.0 & 0.0 & 6.3 \\
& Chlorhexidine & 66.7 & 57.1 & 50.0 \\
Child Health & Antenatal corticosteroids & 83.3 & 57.1 & 31.3 \\
& Resuscitation devices & 66.7 & 71.4 & 84.4 \\
& Amoxicillin* & N/A & N/A & 52.2 \\
\hline
\end{tabular}

* data from $32 \mathrm{CSB}, * *$ data from $23 \mathrm{CSB}, \mathrm{N} / \mathrm{A}$ : not collected

comprehensive or basic emergency obstetric and newborn care or child health during the evaluation.

\section{Basic emergency obstetric and newborn care services}

Availability of BEmONC tracer items at primary care level was very low. On average the CSBs had only about five of the thirteen (38\%) tracer items available on site. Across all basic health facilities surveyed, no staff had been trained in EmONC in the preceding two years. Similarly no national guidelines in BEmONC were available at this level. Availability of magnesium sulfate differed by level of hospital; surprisingly it was low in university hospitals $(16.6 \%)$ but it was more available $(57.1 \%)$ in referral hospitals. One reason for that may be the third level of the referral system of the university hospitals and the ministry of health did not consider the CHU as a priority site for the distribution of magnesium sulfate across the country. Five years ago, the EmONC assessment carried out in all facilities in the country reported that $12.9 \%$ of hospitals and $10.2 \%$ of basic health centers had magnesium sulfate available on $\operatorname{site}^{15}$. We found also that only $6.9 \%$ of the primary care facilities surveyed had magnesium sulfate (unexpired) on site during the assessment. While no progress has been made in supplying magnesium sulfate at CSBs there has been an increase in its availability at referral hospitals.

\section{Comprehensive emergency obstetric and newborn care services}

As expected, district hospitals had the lowest mean score for availability of tracer items for CEmONC services. Even at university hospitals, no facility had all tracer items available. Few regional referral hospitals, two out of nine, and none of the district hospitals had an incubator. Only one out of five district referral hospitals and half of regional referral hospitals had anesthesia equipment. Lack of equipment in district referral hospital may be due to the weak support from the ministry of health to this lower level of public hospitals. There was no major change in availability of functional anesthesia equipment from four years ago when this varied between one third to one half of the hospitals sampled in the EmONC survey ${ }^{15}$. Blood transfusion capacity is low at hospitals. Strengthening the national blood transfusion system is thus still an important challenge for the country, particularly hospitals offering CEmONC services.

\section{Life-saving commodities in maternal, newborn and child health}

Availability of the thirteen key life-saving commodities for prevention and treatment of major causes of maternal, newborn, child death is generally low, except for amoxicillin at primary care facilities and first referral hospitals, and 
newborn resuscitation devices at university hospitals where neonatology and pediatric units are available. Availability of zinc and ORS were present in half of primary care sites assessed. Introduction of misoprostol and chlorhexidine in Madagascar through pilot activities in a few sites explain the lack of these essential drugs across all public health facilities.

Our findings are important to inform program managers, technical partners and donors, as well as officials of the Ministry of Health at district, regional and central levels. Findings about availability of tracer items for maternal, newborn and child services readiness showed gaps that must be overcome by all stakeholders. Even if our survey covered only a sample of health facilities in fifteen regions out of twenty-two nationwide, innovative strategies to improve maternal, newborn and child health services could be derived from its findings and applied in the entire country. The issue of addressing these challenges in a sustainable way should be a major priority of all health programs. It is hoped that the Ministry of Health and other stakeholders will assume a high degree of national ownership and ongoing investment in human, social and physical capital $^{24,28}$. Furthermore, Madagascar can now start to apply the global strategy of the UN Commission on Life-Saving Commodities in relation to the thirteen life-saving commodities for maternal, newborn and child health ${ }^{24}$ that fosters increasing access to and appropriate use at health facilities to address the leading preventable causes of maternal, newborn and child deaths.

A main strength of this assessment was the use of SARA data collection tool adapted to fit our information needs for maternal, newborn and child health services in public facilities. Our approach to service readiness yields objective information about the capacity of health facilities to provide these services. The assessment covered all public health facility types, including basic centers, referral hospitals at district and regional level and university hospitals, to achieve a comprehensive picture of maternal, newborn and child health services in the public sector.

This study has some limitations: A methodological limitation concerns the sampling as the health facilities selected are located in the fifteen USAID intervention regions and did not cover all 22 regions. However, these fifteen regions cover nearly $80 \%$ of the population. Incompleteness of child health tracer item data was a study limitation; nonetheless an overview of management of diarrheal disease among children was obtained for $72 \%$ of basic health centers. This study did not assess health provider skills or delivery of actual services to clients; this was done previously and was not our primary focus ${ }^{27,29}$.

As our results showed an accurate picture of maternal, newborn and child health service status at public health facilities in fifteen USAID intervention regions on the eve of the MDG deadline, this survey can be extended to the remaining seven regions to capture a complete picture of the situation in the country. Efforts by multiple stakeholders are ongoing to meet targets MDG 4 and 5 and Sustainability Development Goals 3.1 and 3.2, and can certainly result in improvement of maternal, newborn and child health if aimed at the areas of greatest need. All facilities need to have high levels of essential items, and wide variations, for example in some items such as newborn resuscitation equipment must be reduced.

\section{Conclusion}

Overall availability of tracer items in maternal, newborn and child health remains low in the public health facilities in Madagascar. Since the findings of this survey were taken into account in the development of the national Roadmap for accelerating the reduction of the maternal and neonatal mortality 2015-2019 in Madagascar, the Ministry of Health should strengthen its leadership and coordinate the support and collaboration by partners and stakeholders to work on the public health facilities 'capacity to deliver services. This will enhance the improvement of maternal, newborn and child health nationwide.

\section{Acknowledgements}

We are grateful to the Ministry of Public HealthMadagascar for their support and for allowing us to conduct this assessment in the public health facilities. We also thank all the health management teams and health facility staff at the regional and 
district levels and the data collectors. This assessment was made possible by the generous support of the American people through the United States Agency for International Development (USAID), under the terms of the Cooperative Agreement AID-OAA-A-14-00028. The contents are the responsibility of the authors and do not necessarily reflect the views of USAID or the United States Government.

\section{Conflict of Interest}

We declare we have no conflict of interest.

\section{Contribution of Authors}

Favero R, Rakotovao J P, Razakariasy R M E, Andriantsimietry S, Raymond R and Bazant $\mathrm{E}$ developed the protocol. Ramiandrison R E, Favero R, Rakotovao J P, Andriantsimietry S analyzed the data. All the authors wrote the manuscript and approved the published version.

\section{References}

1. Hogan MC, Foreman KJ, Naghavi M et al. Maternal mortality for 181 countries, 1980-2008: a systematic analysis of progress towards Millennium Development Goals 5. Lancet 2010; 375:16091623.

2. UNDP. Human Development Report 2003. Millennium Development Goals: a compact among nations to end poverty. Oxford: Oxford University Press.

3. http://www.un.org/sustainabledevelopment/sustainabledevelopment-goals/.(accessed on 01/26/2015).

4. Greco G, Powell-Jackson T, Borghi J, Mills A. Countdown to 2015: assessment of donor assistance to maternal, newborn, and child health between 2003 and 2006. Lancet, 2008; 371:1268-1275.

5. Say L, Chou D, Gemmill A, et al. Global causes of maternal death: a WHO systematic analysis. Lancet Glob Health, 2014; 2: e323-33. http://dx.doi.org/ $10.1016 / \mathrm{S} 2214-109 \mathrm{X}(14) 70227-\mathrm{X}$

6. Campbell OM, Graham WJ. Strategies for reducing maternal mortality: getting on with what works. Lancet 2006; 368 (9543): 1284-1299.

7. Koblinsky M, Matthews Z, Hussein J, Mavalarkar D, Mricha MK, Arwar I et al. Going to scale with professional skilled care. Lancet 2006; 368 (9544): 1377-1386

8. Ronsmans C, Graham WJ. Maternal mortality: who, when, where and why. Lancet 2006; 368 (9542): 1189-1200.

9. The Partnership for maternal, newborn and child health.
(2006). Opportunities for Africa's newborns. http://www.who.int/pmnch/media/publications/oanf ullreport.pdf, accessed on 06/23/2015).

10. WHO, UNFPA, UNICEF, AMDD. Monitoring emergency obstetric care, a handbook. Geneva: WHO,2009.http://www.who.int/reproductivehealth/ publications/monitoring/9789241547734/en/index.h tml (accessed on 10/20/2014).

11. Gabrych S, Zanger P, Campbell O. Emergency obstetric care availability: a critical assessment of the current indicator. Trop Med Int Health, 2012, 17:2-8.

12. WHO: Maternal mortality, factsheet 348 , updated May 2014. http://www.who.int/mediacentre/factsheets/ fs348/en/, (accessed on 02/14/2015).

13. The Lancet Commissions. The Millennium Development Goals: a cross-sectoral analysis and principles for goal setting after 2015. Lancet, 2010: 376: 991 1021.

14. INSTAT Madagascar .Enquête nationale sur le suivi des objectifs du millénaire pour le développement à Madagascar. http://www.instat.mg/pdf/ensomd2012 -2013_resume.pdf (accessed on 03/05/2015).

15. Vice Primature de la Santé Publique. 2010. Evaluation des besoins en matière de soins obstétricaux et néonatals d'urgence à Madagascar. Vice Primature de la Santé Publique, UNFPA, UNICEF, OMS, AMDD, MSIS.http://www.hayzara.org/BANQUEDE-CONNAISSANCES/Sante/Evaluation-desBesoins-en-Matiere-de-Soins-Obstetricaux-etNeonatals-dUrgence-a-Madagascar-Vice-PrimatureChargee-de-la-Sante-Publique-UNFPA-UNICEFOMS-AMDD-MSIS-Mars-2010 (accessed on 03/05/2015).

16. Averting Maternal Death and Disability Program, 2003. AMDD workbook: Using the UN process Indicators of Emergency Obstetric Services. Questions and Answers. AMDD, New York.

17. Maternal and Child Survival Program,http://www. mcsprogram.org / (accessed on 07/14/2015).

18. WHO: Service availability and readiness assessment, an annual monitoring system for service delivery. Reference Manual, version 2.1. Geneva WHO, 2013.http:www.who.int/iris/bitstream/10665/10407 5/1/WHO_HIS_HSI_RME_2013_1_eng.pdf (accessed on 09/10/2014).

19. INSTAT Madagascar. (2013). Madagascar en chiffres, population et démographie. http://www.instat.mg/ index.php?option=com_content $\& v i e w=$ article $\&$ id $=$ 33\&Itemid=56. $($ Accessed on 02/10/ 2015).

20. JSI/MAHEFA.http://www.jsi.com/JSIInternet/Intl Health/project/display.cfm?ctid=na\&cid=na\&tid=40\&id $=8561($ accessed on $07 / 14 / 2015)$.

21. MSH/MIKOLO. http://www.msh.org/ourwork/projects/madagascar-mikolo (accessed on 07/14/2015).

22. O’Neill K, Takane M, Sheffel A, Abou-Zahr C, Boerma T. Monitoring service delivery for universal health coverage: the Service Availability and Readiness Assessment. Bull World Health Organ 2013; 91: 
923-931.Doi:http://dx.doi.org/10.2471/BLT.12 .116798.CommCare.https://www.commcarehq.org/ (accessed on 08/20/2014).

23. UN commission on life-saving commodities for women and children. Commissioner`s report. September 2012, UN every woman, every child, New York. http://www.unfpa.org/webdav/site/global/shared /images/publications/2012/Final\%20UN\%20Commi ssion\%20Report_14sept2012.pdf (accessed on 10/27/2014).

24. Quality of care of the Prevention and Management of Common Maternal and Newborn Complications in Health Facilities in Madagascar. MCHIP Madagascar- Jhpiego Baltimore, December 2011. http://www.mchip.net/sites/default/files/Madagascar \%20QoC\%20report\%20-\%20Final.pdf (accessed on 03/05/2015).

25. Duley L. Maternal mortality associated with hypertensive disorders of pregnancy in Africa, Asia,Latin America and the
Carribean. BJOG 1992; 99(7):547-553.

26. Bazant E, Rakotovao JP, Rasolomanana JR, Tripathi V, Gomez P, Favero R et al. Qualité des soins pour prévenir et traiter l'hémorragie du postpartum et la pré-éclampsie/ éclampsie: une évaluation fondée sur l'observation dans les hôpitaux de Madagascar. Med Sante Trop, 2013; 23 (2) : 168-75. Doi : 10.1684/mst.2013.0161.

27. Waage J, Banerji R, Campbell O, Chirwa E, Collender G, Dieltiens V et al. The Millenium Development Goals: a cross-sectoral analysis and principles for goal setting after 2015. The Lancet Commissions; 2010, 376. http://www.thelancet.com.

28. Ranjalahy R, Bazant E, Rakotovao JP, Cantor D, JengJoof Y, Noeliarivelo, A et al. Evaluation de la qualité des soins maternels et néonatals à Madagascar. Maternal and Child Health Integrated Program (MCHIP). Johns Hopkins University, TANDEM, Jhpiego, Baltimore, 2011. 